\title{
Effects of Treadmill Training and Stationary Cycling Training to Improve Ambulatory Function and Cardiovascular Fitness in Hemiparetic Stroke Patients
}

\author{
MIAN ALI RAZA ${ }^{1}$, MISBAH WARIS ${ }^{2}$, FARRUKH MURTAZA $^{3}$, SADAF WARIS $^{4}$, RABIYA NOOR $^{5}$, SALMAN BASHIR $^{6}$ \\ ${ }^{1}$ Senior Lecturer, University Institute of Physical Therapy, University of Lahore \\ ${ }^{2}$ Senior Lecturer, Riphah International University, Lahore \\ ${ }^{3}$ Senior Lecturer, University Institute Of Physical Therapy, University Of Lahore \\ ${ }^{4}$ Demonstrator, University of Management and Technology, Sialkot Campus \\ ${ }^{5}$ Assistant Professor, Riphah International University, Lahore \\ ${ }^{6}$ Professor, Riphah International University, Lahore \\ Correspondence to Dr. Sadaf Waris, Email: dr.sadaf54@gmail.com, Contact No:0308-8112501
}

\begin{abstract}
Background: Stroke is a universal health care disease, the important cause of long time disability in world. Stroke leads to an inactive living, physical restrictions, and not good physical levels, which are related with common poststroke participation limits. Aerobic capability and walking ability are decreased in old chronic patients of stroke.

Aim: To determine the effects of treadmill training and stationary cycling training to improve ambulatory function and cardiovascular fitness in hemiparetic stroke patients

Methods: 54 chronic stroke patients were allocated to treadmill training group( $n=27)$ or stationary cycle exercise group $(n=27)$. All participants received conventional physical therapy along with treadmill or stationary cycle training. The 10MWT was conducted to measure gait function and 6 min walk test was used to measure cardiovascular health.

Results: The mean of treadmill group for 10 meter walk test is $10.01+15.48$. The mean of stationary cycle group for 10 meter walk test is $9.80+6.77$. The mean of treadmill group for 6 min walk test is $22.04+17.45$. The mean of stationary cycle group for 6 min walk test is $23.20+22.92$. The $p$ value of 0.000 shows significant difference. This significant difference reflects that both interventions show equal improvement in participants. There is significant difference between pre and post treatment values of both interventions. Both intervention groups displayed significant effect in ambulatory functions and cardiovascular fitness. The results between groups were non significant, it means both interventions showed equal effect but results with in groups were significant.

Conclusion: The study showed that treadmill training and stationary cycling training equally enhanced the gait ability and cardiovascular health of chronic stroke patients. Therefore, these exercises could be used to enhance walking and cardiovascular health in management of stroke.
\end{abstract}

Keywords: treadmill training, stationary cycle training, ambulatory function, cardiovascular fitness, stroke

\section{INTRODUCTION}

Stroke is a universal health care issue, the top most cause of constant disability in world. The common causes after stroke such as muscle weakness, balance, cognitive impairments and hemiparesis. Stroke prompts a stationary way of life, physical dormancy, and not great wellness levels, which are connected with regular post-stroke practical cutoff points. Aerobic limit and walking are decreased in old patients with constant stroke and that $\mathrm{VO}_{2}$ estimations of under $20 \mathrm{~mL} / \mathrm{min}$ are related with restricted physical capacity when performing day by day exercises ${ }^{1}$.

The treadmill has been used in clinical and hospital settings for testing patients with pulmonary and cardiac disorders. The potential musculoskeletal benefits of the treadmill on patient's performance is just start to receive the care of investigators. positive effects on gait have been shown when exercises help joint movements of the legs, devices such as the treadmill investigation ${ }^{2}$.

Stationary cycling, has been utilized for preparing patients with or without sensory system issue, which requires less balance ability, trouble in keeping up

Received on 17-04-2021

Accepted on 29-08-2021 equilibrium and autonomous gait. Cycling and walking development examples of joint flexion and expansion developments and unpredictable muscle contraction. Cycling can improve practical versatility, strolling and work out. Stationary cycling exercise utilized with numerous different interventions in the clinical. The impacts of cycling intervention on the walk and balance of patients of stroke ${ }^{3}$.

In the world, one of the prime source of functional restriction is stroke. People suffered a stroke experience restriction such as a reduce ability of gait, and decreased participation in ADLs. These disabilities are often related to the hemiparesis that occurs after stroke. The degrees of restrictions related with stroke bring about a monetary weight. Perceiving techniques to augment work and diminish the weight related with stroke is of most extreme significance. In the early not many weeks after a stroke, neurological deformities may improve because of neuro versatility. This mind pliancy may result from enrollment of various pathways, assistance of existing however lethargic synaptic intersections, abortization of dendrites and synaptogenesis. The power of holding contrasts extraordinarily among people with comparative clinical seriousness in the intense stage. The comprehension of the instruments which forestall or elevate recuperation is basic to propel the executive's methodologies and amplify 
work. During treatment, tactile criticism and engine action is obligatory. Various investigations have point a connection between afferent incitement and changes in neural movement, for example, redundant, successful objective situated action, and FES. Impacts because of stroke can possibly be expanded through treatment concentrating on improving weaknesses, practical impediments and limitations of movement interest. One such strategy that may address these results is cycling with/without ES which can possibly improve engine limit and proposed bring about more noteworthy interest and action execution after a stroke ${ }^{4}$.

An essential goal in present stroke restoration is on upgrade strolling limit and step related exercises, be that as it may, most recovery treatment centering locomotor capacity uses practice well underneath vigorous forces vital for improving cardiorespiratory wellness. Studies has results that vigorous limit is associated with strolling velocity and separation, referencing the significance of a preparation methodology that objectives improving oxygen consuming limit. The hypothesis behind recovery intended to improve locomotor results is engaged around the dreary idea of the developments, which is believed to be a power behind practical improvement. One preparing methodology that has not gotten as a lot of consideration in people with interminable stroke is the utilization of stationary cycling, with or without (FES) help. Cycling creates elective monotonous developments that require appropriately planned intra-and between strong composed withdrawal between the flexors and extensors of the hip, knee, and lower leg which is additionally required during strolling ${ }^{5}$.

Body-weight-supported treadmill preparing (BWSTT) permit innocuous strolling practice in beginning phases of cardiovascular recuperation via naturally counterbalancing an extent of weight. As a walk retraining methodology body weight upheld treadmill preparing (BWSTT) is extended with the estimations of action subordinate practice, power and errand explicitness. the viability of body weight upheld treadmill preparing (BWSTT) on walking job in the subacute post stroke period ${ }^{6}$.

Stationary cycling can possibly be acted in the home by slight help from others, and can give reliable practice conditions that permit a high number of cycles. Stationary cycling has been explored in restoration programs and joint leg exercise to strolling while the regular of the patient's body weight is upheld by the seat ${ }^{7}$.

That supports a natural basis for treadmill (TM) as an undertaking focused preparing model to support locomotor improvement, giving an oxygen consuming activity improves cardiovascular-metabolic wellbeing and wellness in the stroke. practice modalities have shown to increment cardiovascular wellness. Hemiparetic step evenness with treadmill (TM) is striking and is well-portrayed for both the body weight upheld (BWST) and self-bolstered TM conditions ${ }^{8}$.

Now a day, the treadmill speed has been considered as an important clue to enhance the gait ability of stroke patients. The conventional treadmill training speed that has been accepted universally in patients with stroke is slow. Fast walking on the treadmill has been showed to improve gait parameters significantly compared to walking slowly. Despite the widespread use of treadmill training, it has restrictions in using compensation strategies rather than recovery of normal kinetic symmetry. Therefore, stimulation that produce sensory messages from cutaneous or muscle proprioceptive receptors is needed to progress the use of the hemiparetic side instead of excessive use of the less affected side ${ }^{9}$.

This study conducted to proof the effectiveness of treadmill intervention and stationary cycling training to improve ambulatory function and cardiovascular fitness in stroke patients. This study proposed the best aerobic training for stroke patients to improve ambulatory function and cardiovascular fitness.

\section{MATERIAL AND METHODS}

This was a quasi-experimental study. 54 chronic hemiparetic stroke patients were allocated to treadmill training group $(n=27)$ and stationary cycle exercise group $(n=27)$. All participants received conventional physical therapy along with treadmill or stationary cycle training. The 10 meter walk test was conducted to measure gait function and 6 min walk test was used to measure cardiovascular health. Study was completed in 6 months after the approval of synopsis. Both male and female patient having stroke from more than 6 months were selected. Patients have enough cognition level and walking speed. Patients are not involved in any other exercise related to mobility. Patients having other disease or disability that makes walk training impossible and stroke patients with vision and hearing problems were excluded. The data was collected from Chaudhary Muhammad Akram Teaching and Research Hospital Lahore. Using SPSS v.23 the data was managed and analyzed. The continuous variables expressed as mean \pm S.D. whereas qualitative variables expressed in the form of frequency table and percentages.

\section{RESULTS}

Table 1 shows the summary of total participants according to gender, post stroke duration and effected side. The total number of participants who participated in this study were 50.

Table 2 shows the gender distribution of participants who received either treadmill training or stationary cycle training. 25 participants received treadmill training and 25 participants received stationary cycle training. In treadmill training group all the 25 participants were male. In stationary cycle group 18 participants were male and 7 participants were female.

Table 3 shows the frequency of effected side of the participants. In treadmill intervention group 20 participants have left side hemiplegia and 5 participants were with right side hemiplegia. In stationary cycle intervention group 19 participants have left side hemiplegia and 6 participants were with right side hemiplegia.

Table 4 shows the mean \pm S.D of both interventional groups for between group analysis of 10 meter walk test and 6 min walk test. The mean of treadmill group for 10 meter walk test is $10.01 \pm 15.48$. The mean of stationary cycle group for 10 meter walk test is $9.80 \pm 6.77$. The mean of treadmill group for 6 min walk test is $22.04 \pm 17.45$. The 
mean of stationary cycle group for 6 min walk test is $23.20 \pm 22.92$.

Table 5 shows the significant difference of pre and post treatment values for both interventional groups. This significant difference reflects that both interventions show equal improvement in participants. There is significant difference between pre and post treatment values of both interventions.

Table 1: Case Processing Summary

\begin{tabular}{|l|c|c|c|c|c|c|}
\hline \multirow{2}{*}{} & \multicolumn{3}{|c|}{ Cases } & \multicolumn{2}{c|}{ Total } \\
\cline { 2 - 7 } & \multicolumn{2}{|c|}{ Valid } & \multicolumn{2}{c|}{ Missing } & Percent & N \\
\cline { 2 - 7 } & $\mathbf{N}$ & Percent & N & Percent \\
\hline Gender * intervention type & 50 & $100.0 \%$ & 0 & $0.0 \%$ & 50 & $100.0 \%$ \\
\hline Post stroke duration * intervention type & 50 & $100.0 \%$ & 0 & $0.0 \%$ & 50 & $100.0 \%$ \\
\hline Effected side * intervention type & 50 & $100.0 \%$ & 0 & $0.0 \%$ & 50 & $100.0 \%$ \\
\hline
\end{tabular}

Table 2: Gender * intervention type Cross tabulation

\begin{tabular}{|l|c|c|c|}
\hline \multirow{2}{*}{ Gender } & \multicolumn{2}{|c|}{ Intervention type } & \multirow{2}{*}{ Total } \\
\cline { 2 - 3 } & Treadmill & Stationary cycle & 43 \\
\hline Male & 25 & 18 & 7 \\
\hline Female & 0 & 7 & 50 \\
\hline Total & 25 & 25 & \\
\hline
\end{tabular}

Table 3: Effected side * intervention type Cross tabulation

\begin{tabular}{|l|c|c|c|}
\hline \multirow{2}{*}{$\begin{array}{l}\text { Effected } \\
\text { side }\end{array}$} & \multicolumn{2}{|c|}{ Intervention type } & \multirow{2}{*}{ Total } \\
\cline { 2 - 3 } & Treadmill & Stationary cycle & 39 \\
\hline left & 20 & 19 & 11 \\
\hline right & 5 & 6 & 50 \\
\hline Total & 25 & 25 & \\
\hline
\end{tabular}

Table 4: Group statistics

\begin{tabular}{|l|c|c|c|c|c|}
\hline & Intervention type & N & Mean & Std. Deviation & Std. Error Mean \\
\hline \multirow{2}{*}{ 10meter_deffrence } & Treadmill & 25 & 10.0160 & 15.48195 & 3.09639 \\
\cline { 2 - 6 } & stationary cycle & 25 & 9.8092 & 6.77310 & 1.35462 \\
\hline \multirow{2}{*}{ 6min_deffrence } & Treadmill & 25 & 22.0400 & 17.45346 & 3.49069 \\
\cline { 2 - 6 } & stationary cycle & 25 & 23.2000 & 22.92742 & 4.58548 \\
\hline
\end{tabular}

Table 5: Paired Samples Correlations

\begin{tabular}{|l|ll|c|c|c|}
\hline Pair 1 & $\begin{array}{l}\text { G1-10meterpre \& G1- } \\
10 \text { meterpost }\end{array}$ & $\mathbf{n}$ & Correlation & Sig. \\
\hline Pair 2 & $\begin{array}{l}\text { G1-6minpre \& G1- } \\
\text { 6minpost }\end{array}$ & 25 & .959 & .000 \\
\hline Pair 3 & $\begin{array}{l}\text { G2-10 meterpre \& G2- } \\
10 \text { meterpost }\end{array}$ & 25 & .971 & .000 \\
\hline Pair 4 & $\begin{array}{l}\text { G2-6minpre \& G2-6 } \\
\text { minpost }\end{array}$ & 25 & .882 & .000 \\
\hline
\end{tabular}

\section{DISCUSSION}

This study investigated the impact of treadmill training and stationary cycle training on ambulatory function and cardiovascular fitness in stroke patients in the chronic phase. The results of this study reflect that treadmill training and stationary cycle training in combination with traditional physiotherapy improved the ambulation and cardiovascular status of patients with hemiplegia. Ambulatory function was measured by using 10 meter walk test and cardiovascular fitness was assessed by using 6 min walk test. The cardiovascular fitness and ambulatory function of the participants improved in both interventional groups.

Recent study applied six-week interventional program to stroke patient who were divided in two groups in order to investigate the effect of each exercise on ambulatory function and cardiovascular fitness. One group received treadmill training with conventional physiotherapy and other group received stationary cycling training with traditional physiotherapy.

In this study ambulatory function measured 10-MWT significantly improved in both treadmill group and stationary cycle group. As well as cardiovascular fitness measured by 6-MWT significantly improved in both treadmill training group and stationary cycle group. Both intervention group showed improvement in 10-MWT and 6-MWT. Results of this study showed that both groups have equal improvement in ambulation and cardiovascular status.

As results show that both treatment groups have equal effects on patients. Treadmill group showed improvement in both 10-MWT and 6-MWT. Stationary cycle group also showed equal improvement in both 10-MWT and 6-MWT. On the other perspective difference is not only seen between group but also with in the groups. Both treatment groups showed improvement in pre and post treatment values. There is significant difference between pretreatment and post treatment values of 10-MWT and 6MWT. The paired sample t-test displayed the $0.000 \mathrm{P}$ value. The treadmill group showed improvement in pre and post treatment value of 10-MWT and 6-MWT. Stationary cycle group also showed improvement in pre and post treatment value of 10-MWT and 6-MWT.

Another perspective of this study is that combination therapy is more effective for the rehabilitation of stroke patients. Conventional physical therapy in addition of aerobic exercise is more effective in stroke patients to improve their functional independence. As we discussed both treatment groups in this study showed improvement in ambulation and cardiovascular status. So combination therapy will be more effective to improve aerobic capacity of patient.

The findings of another study suggest that cycling and walking share comparative locomotor examples of alternative equal flexion and expansion movements. The results of this study showed significant difference in 6MWT from baseline to week $8(p<0.03)$. Current study also showed similar results with $p$ value of 0.000 . Henceforth, stationary cycling exercise, which utilizes proportional development of the lower appendages and requires coordination of relating muscles, viably expanded the stride capacity. Cycling preparing animates engine locales in the 
focal sensory system and enacts the cerebral cortex which in the long run improves cognitive learning and equilibrium. This impact absolutely applies to ceaseless stroke patients. Comparative outcomes are appeared by our examination that cycling and strolling have beneficial outcomes to improve lower limb co-ordination ${ }^{4}$.

The noticed developments in cardiovascular health may be related to advancement in cognitive function. Another research shows the significant difference $(p<$ 0.05 ) in 10MWT after treadmill intervention. These results are similar to current study with $p$ value of 0.000 in 10MWT.Other researches have shown that aerobic limit or peak oxygen utilization and strolling execution are decreased in old stroke patients and that low oxygen utilization levels are related with constrained physical capacity when performing every day exercises. Be that as it may, perseverance practice advantageously influences wandering pulse. In this manner, expanding the wellness levels of stroke patients may turn away after stroke issues including weariness, expanded vitality cost of mobility, falls and breaks, and low life quality ${ }^{5}$.

Results of this study matched with other study. This study conducted in 2015. This study indicates the nonsignificant difference between two interventional groups. They suggest that stationary cycle and treadmill are equally effected in stroke patients to improve balance. Similar results are showed by our study that treadmill training and stationary cycle training have same effects for hemiplegic patients to improve ambulation and cardio fitness ${ }^{6}$.

Treadmill intervention enhanced walking abilities in hemiparetic patients. These findings are matched to previous studies. Treadmill exercise with or without suspension improves walking abilities. The results of our study also showed that treadmill intervention have positive effects on gait training ${ }^{7}$.

A study conducted in 2016, outcomes of this study showed small to moderate effect sizes favoring the aerobic exercise group: 6 -minute walk $(\mathrm{g}=0.366, \mathrm{P}<0.001)$ and 10 -meter walk ( $g=0.411, P=0.002)$, while the up-n-go test was not significant $(g=-0.150, P=0.330)$. These results are similar to current study. In current study significant difference reflects that both interventions show equal improvement in participants. There is significant difference between pre and post treatment values of both interventions. $P$ value of both interventions are $<0.001^{10}$.

Another study conducted in 2017, the findings of this study showed that individuals after stroke who get treadmill preparing with or without body weight support are not bound to improve their capacity to walk freely contrasted and individuals after stroke not getting treadmill preparing, yet strolling velocity and strolling continuance may improve. The results of this study matched with current study because significant value of 10 meter walk test for both interventional groups is 0.090 . And significant value of 6 min walk test is .896. The results show that both interventional groups have equal effects ${ }^{11}$.

In conclusion, this study reflects that treadmill training and stationary cycling training enhanced the ambulatory capacity and cardiovascular status of patients with hemiplegia in the chronic phase. So that, these exercises could be used to enhance ambulation capacity and cardiovascular status in stroke management.

\section{CONCLUSION}

The study concluded that treadmill training and stationary cycling training equally improved the ambulatory function and cardiovascular fitness of patients with stroke in the chronic phase. Therefore, these exercises could be used to improve walking ability and cardiovascular health in stroke rehabilitation.

\section{Conflict of interest: Nil}

\section{REFERENCES}

1. Brogårdh $\mathrm{C}$, Lexell $\mathrm{J}$. Effects of cardiorespiratory fitness and muscle-resistance training after stroke. PM\&R. 2012;4(11):901-7.

2. Abu-Saifan S. Social entrepreneurship: definition and boundaries. Technology innovation management review. 2012;2(2).

3. Kim SJ, Cho HY, Kim YL, Lee SM. Effects of stationary cycling exercise on the balance and gait abilities of chronic stroke patients. Journal of physical therapy science. 2015;27(11):3529-31.

4. Shariat A, Najafabadi MG, Ansari NN, Cleland JA, Singh MAF, Memari A-H, et al. The effects of cycling with and without functional electrical stimulation on lower limb dysfunction in patients post-stroke: a systematic review with meta-analysis. NeuroRehabilitation. 2019;44(3):389-412.

5. Aaron SE, VanDerwerker CJ, Embry AE, Newton JH, Lee SC, Gregory CM. FES-Assisted Cycling Improves Aerobic Capacity and Locomotor Function Post-CVA. Medicine and science in sports and exercise. 2018;50(3):400.

6. MacKay-Lyons M, McDonald A, Matheson J, Eskes G, Klus $M-A$. Dual effects of body-weight supported treadmill training on cardiovascular fitness and walking ability early after stroke: a randomized controlled trial. Neurorehabilitation and neural repair. 2013;27(7):644-53.

7. Prosser LA, Stanley CJ, Norman TL, Park HS, Damiano DL. Comparison of elliptical training, stationary cycling, treadmill walking and overground walking. Electromyographic patterns. Gait \& posture. 2011;33(2):244-50.

8. Macko R, Ivey F, Forrester L. Task-oriented aerobic exercise in chronic hemiparetic stroke: training protocols and treatment effects. Topics in stroke rehabilitation. 2005;12(1):45-57.

9. Choi W, Han D, Kim J, Lee S. Whole-body vibration combined with treadmill training improves walking performance in post-stroke patients: a randomized controlled trial. Medical science monitor: international medical journal of experimental and clinical research. 2017;23:4918.

10. Kendall BJ, Gothe NP. Effect of aerobic exercise interventions on mobility among stroke patients: a systematic review. American Journal of Physical Medicine \& Rehabilitation. 2016;95(3):214-24.

11. Mehrholz J, Thomas S, Elsner B. Treadmill training and body weight support for walking after stroke. Cochrane Database of Systematic Reviews. 2017(8). 